\title{
MOVILIDAD DE INGRESOS Y MOVIMIENTOS DEMOGRÁFICOS EN AMÉRICA LATINA *
}

INCOME MOBILITY AND DEMOGRAPHIC MOVEMENTS IN LATIN AMERICA

\author{
Martin Trombetta*
}

enviado: 15 marzo 2017- aceptado: 3 mayo 2017

\begin{abstract}
Resumen
En este trabajo contribuimos al estudio de la movilidad de ingresos de corto plazo en América Latina computando la importancia de los movimientos demográficos en las fluctuaciones del ingreso familiar. Explotando el esquema rotativo de las encuestas de hogares de siete países latinoamericanos, construimos paneles cortos que permiten cuantificar el tamaño y relevancia de los cambios de composición de los hogares en ventanas temporales anuales. Encontramos evidencia de un peso pequeño pero significativo de los movimientos demográficos como factor explicativo de la movilidad de ingresos en el corto plazo. Adicionalmente, exploramos la existencia de estrategias de los hogares para suavizar la evolución de su ingreso per cápita ante movimientos demográficos que se pueden interpretar como shocks exógenos.
\end{abstract}

Código JEL: J10, J13, D31.

Palabras clave: movilidad, inestabilidad, ingresos, demográficos.

\begin{abstract}
In this paper, we contribute to the study of short run income mobility in Latin America by assessing the importance of demographic movements in household income fluctuations. We exploit the rotating scheme of household surveys from seven Latin American countries to build short panels that allow us to quantify the size and

* Universidad Nacional de General Sarmiento. Correo electrónico: martintrombetta@gmail.com

Este trabajo fue galardonado con una mención especial en la octava edición del Congreso Nacional de Estudiantes de Postgrado en Economía (CNEPE) 2017.

Trombetta, M. (2017). Movilidad de ingresos y movimientos demográficos en América Latina. Estudios económicos, 34 (69), 47-70.
\end{abstract}


relevance of household compositional changes in yearly time windows. We find evidence of a small but significant weight of demographic movements as an explicative factor of short-term income mobility. Additionally, we explore the existence of household strategies to smooth the evolution of per capita income as a response to demographic shocks, consisting of entrance to or exit from the labor market.

JEL Code: J10, J13, D31.

Keywords: Mobility, Instability, Income, Demographic.

\section{INTRODUCCIÓN}

El análisis de los movimientos de los ingresos de los hogares en el tiempo ha capturado la atención de una parte considerable de la literatura especializada en los últimos años. En Beccaria et al. (2015), se cuantifica el tamaño de los movimientos de corto plazo en los ingresos de los hogares en América Latina, interpretando esto como una medida de la variabilidad o inestabilidad de ingresos en esta región. Si asumimos que los hogares son adversos al riesgo, la inestabilidad de ingresos constituye una considerable pérdida de bienestar, que resulta particularmente relevante si se tiene en cuenta que los mercados de crédito están poco desarrollados en estos países, de modo que existen escasos mecanismos que permitan suavizar las fluctuaciones.

En este trabajo proponemos avanzar en la línea anterior evaluando la relevancia de los movimientos demográficos en el nivel de variabilidad observada en los ingresos de los hogares. Definimos como movimientos demográficos a todos aquellos cambios de tamaño o composición que un hogar puede experimentar a partir de llegadas de nuevos miembros, salidas de miembros anteriores o la combinación de ambas. Estos pueden ser fruto de fenómenos biológicos (nacimientos o fallecimientos), fenómenos migratorios, fusiones de hogares previamente existentes (frecuentemente, aunque no exclusivamente, debido a matrimonios), división de un hogar en otros varios, entre otras razones. La relevancia de estos movimientos en la evolución temporal del ingreso del hogar responde a varias razones.

En primer lugar, un porcentaje elevado de los miembros que entran o salen de un hogar son perceptores de ingresos, ya sea por su condición de ocupados o bien porque reciben ingresos no laborales de algún tipo (principalmente, transferencias de origen público), de modo que el ingreso total del hogar es afectado automáticamente por estos movimientos. En segundo lugar, aun si los movimientos 
demográficos no involucran a perceptores de ingresos, sí alteran las necesidades de consumo del hogar como unidad económica, lo que indica que es preciso analizar la evolución del ingreso per cápita del mismo. En tercer lugar, los cambios de composición del hogar factiblemente afectan el mecanismo de distribución del ingreso intrahogar (sharing rule) a partir de las diferentes características de los integrantes (por ejemplo, es concebible que gastos asociados al consumo de niños pequeños sean priorizados por sobre los de otros miembros). Por último, y como consecuencia de lo anterior, es posible que las estrategias de los individuos (en particular, en lo que respecta a participación en el mercado de trabajo) sean sensibles al tamaño y composición del hogar que integran.

Siguiendo la línea de trabajos anteriores, explotamos el esquema rotativo de las encuestas de hogares de siete países latinoamericanos para construir paneles anuales que permiten observar y descomponer los cambios del ingreso del hogar en ventanas temporales de un año. La muestra de países empleada contiene a Argentina, Brasil, Costa Rica, Ecuador, México, Paraguay y Perú y cubre el período 2003-2015. Utilizamos esta base de datos para cumplir varios objetivos: cuantificar el tamaño y la frecuencia de los movimientos demográficos, computar su contribución a las fluctuaciones de ingresos observadas y analizar la existencia de estrategias al interior de los hogares que permitan suavizar su impacto.

El resto del trabajo se encuentra estructurado de la siguiente manera. La sección 2 provee una breve revisión de la literatura específica. La sección 3 repasa la metodología seguida por trabajos anteriores para medir la variabilidad de ingresos en bases de datos de este tipo e indica las fuentes de información utilizadas. La sección 4 presenta los resultados de diversos ejercicios realizados con el fin de computar la contribución de los movimientos demográficos a la movilidad observada. La sección 5 analiza la existencia de estrategias al interior de los hogares que pueden ser interpretadas como respuestas a estos movimientos. Finalmente, la sección 6 provee algunas conclusiones.

\section{REVISIÓN DE LA LITERATURA}

Existe abundante literatura que enfatiza el rol del hogar como unidad perceptora de ingresos en lugar del individuo. Si bien son los individuos quienes reciben directamente ingresos, sea laborales (trabajando para una empresa o para sí mismos) o no laborales (a través de derechos de propiedad sobre activos o como beneficiarios de transferencias), existe un consenso general sobre la idea de que este ingreso es distribuido dentro del hogar de acuerdo con reglas que generalmen- 
te dependen de las preferencias de más de uno de los miembros. La modelización más habitual de este problema de optimización del uso de los recursos al interior del hogar es provista por el trabajo pionero de Chiappori (1992). En este marco de análisis, la solución al problema puede interpretarse en términos de la existencia de una regla de asignación del ingreso intrahogar (sharing rule). Los hallazgos empíricos presentados aquí son consistentes con esta formulación del problema aunque no se restringen a ella y también resultan compatibles con otras estrategias posibles de modelización.

Existe una abundante literatura que analiza la movilidad de ingresos de corto plazo en términos de inestabilidad, volatilidad o inseguridad de ingresos. Sin embargo, el espectro de trabajos empíricos que analizan la importancia de los movimientos demográficos en la determinación del sendero temporal de ingreso de los hogares es más bien reducido. A continuación, analizamos los principales aportes existentes en la literatura.

Duncan y Morgan (1981) proveen uno de los primeros análisis de este vínculo a partir de datos de la Panel Study of Income Dynamics (PSID) para Estados Unidos. Los autores proveen evidencia de una considerable relevancia de los cambios de composición de los hogares en la dinámica de ingresos, al punto de señalar que "las personas pueden tener mayor control de su status económico a través de decisiones sobre matrimonio, divorcio, procreación, duplicando o reduciendo a la mitad los cambios experimentados a partir de acciones tales como buscar un mejor empleo o más trabajo o invertir en su propio capital humano". En otras palabras, aquí aparece la hipótesis de que los cambios de composición del hogar pueden ser endógenos en naturaleza y en sí mismos poseer un objetivo de dinámica de ingresos. Sin embargo, los autores aclaran que algunos movimientos pueden ser de naturaleza exógena, discusión que retomamos en la sección 5. Los resultados de Duncan y Morgan indican que una parte relevante de los cambios observados en los ingresos de los hogares en períodos cortos de tiempo responden a cambios de composición de los hogares. Estos cambios tienen un efecto muy grande sobre el ingreso (tanto en términos absolutos como en términos per cápita) de algunos hogares, especialmente en aquellos donde el jefe es mujer.

Los resultados de Fritzell (1990) coinciden con los anteriores. En este trabajo, el autor analiza la movilidad de ingresos en la economía sueca y compara sus resultados con los de Duncan y Morgan. Nuevamente la evidencia presentada indica que los cambios demográficos tienen un peso importante en los cambios en el ingreso del hogar y, al igual que en Estados Unidos, el impacto es mayor en los hogares con jefes de sexo femenino (aunque el tamaño de esta brecha se reduce 
considerablemente si la mujer tiene hijos, debido a la existencia de políticas de protección social para niños en Suecia). En particular, el trabajo señala que los cambios de composición del hogar pueden tener un impacto mayor sobre el ingreso futuro del hogar que otros determinantes de orden más estructural como la edad o nivel educativo de los miembros.

Los hallazgos originales de Duncan y Morgan coinciden en líneas generales con los de trabajos posteriores que utilizan nuevamente los datos de la PSID a lo largo de un horizonte temporal mayor. En particular, Gittleman y Joyce (1999) reportan resultados análogos luego de incorporar las sucesivas ondas del relevamiento hasta 1991. Estos autores encuentran efectos similares del cambio de composición del hogar, aunque la variable dependiente que utilizan no es el cambio en el ingreso, sino la probabilidad de experimentar un aumento en la posición dentro de la distribución del ingreso de cada período.

La contribución de Jarvis y Jenkins (1997) se centra en un tipo particular de movimiento demográfico, la división de un hogar producto de un divorcio o separación. Los autores utilizan información proveniente de la British Household Panel Survey (BHPS) y concluyen que este tipo de movimientos tiene un fuerte impacto sobre el ingreso de mujeres y niños aunque no tanto así para los varones adultos. Asimismo, analizan brevemente el impacto de estos movimientos sobre la participación laboral de varones y mujeres por separado. Sus resultados indican que tanto hombres como mujeres reducen su participación laboral debido a la división del hogar y la magnitud de la caída es relativamente mayor en mujeres. Sin embargo, este efecto es en buena medida fruto del esquema de protección social existente.

Por su parte, Bartels y Bonke (2013) enfatizan el rol de los hogares como estructuras de distribución del riesgo (risk-sharing): si los shocks de ingresos que sufren los miembros de un hogar son, hasta cierto punto al menos, independientes entre sí, entonces la distribución del ingreso al interior del hogar actúa como un mecanismo de seguro contra los shocks idiosincráticos negativos. Para medir este efecto, utilizan encuestas de hogares longitudinales de Alemania y Gran Bretaña para comparar la varianza de los ingresos individuales con la de los ingresos del hogar. Sus resultados indican que tanto el componente permanente como el transitorio de la varianza son menores cuando se analizan los ingresos de los hogares, lo que los lleva a concluir que, efectivamente, la distribución del ingreso al interior del hogar cumple un rol en la reducción del riesgo agregado que este enfrenta.

Partiendo de un marco de análisis similar, Alves y Martins (2014) retoman el análisis del impacto de los cambios demográficos sobre los cambios del ingreso 
del hogar y encuentran, al igual que los trabajos anteriores, que este efecto tiene un tamaño considerable usando datos tanto de Portugal como de los restantes países de la Unión Europea. Como se puede observar, se trata de un hallazgo general en la literatura para países desarrollados, sin embargo, no existen hasta el momento trabajos que reproduzcan este análisis para países de América Latina. El resto de este trabajo se aboca precisamente a ese objetivo.

\section{DATOS Y METODOLOGÍA}

Perú es el único país de América Latina que realiza una encuesta de hogares de tipo longitudinal. En los restantes países, construimos paneles explotando el esquema rotativo de las encuestas de hogares convencionales. Dado que no todas las encuestas tienen cobertura nacional, y atendiendo a las diferencias que suelen existir entre los mercados de trabajo urbano y rural, restringimos el análisis a las áreas urbanas. Para deflactar los ingresos relevados, se utilizó el índice de precios al consumidor oficial de cada país, excepto para el caso de la Argentina donde la manipulación de estadísticas obliga a utilizar un índice de precios construido a partir de promedios de índices provenientes de institutos de estadísticas provinciales que mantuvieron relevamientos confiables. De este modo, construimos el pool de paneles más grande de que tengamos conocimiento para la región ${ }^{1}$.

Por otra parte, la metodología utilizada para medir la intensidad de la variabilidad de ingresos es fuertemente dependiente del tipo de información estadística disponible. Dado que las encuestas con que contamos visitan a los hogares a lo largo de una ventana temporal reducida, y con el fin de poder realizar un análisis comparativo entre los países de la muestra y otros reportados en la literatura, construimos para cada país todos los paneles posibles de dos observaciones separadas por un año y luego agregamos esos paneles en un pool de paneles para cada país. Por lo expuesto anteriormente, consideramos el hogar como unidad receptora de ingresos pero veremos luego que el análisis de los ingresos individuales será relevante para ciertos ejercicios.

Para medir el grado de inestabilidad de ingresos observado recurrimos al indicador de Fields y Ok (1999), de uso habitual en la literatura:

1 Para mayor detalle sobre el origen de la información utilizada y el proceso de elaboración de la base de datos, ver Beccaria et al. (2015). 


$$
m\left(y_{1}, y_{2}\right)=\frac{1}{n} \sum_{i=1}^{n}\left|\ln y_{2 i}-\ln y_{1 i}\right|
$$

Donde $y_{1}$ e $y_{2}$ son los vectores de ingresos de los hogares en cada observación y $n$ es el tamaño de la muestra. Como se puede apreciar, este indicador mide el tamaño medio de los cambios relativos de ingresos, independientemente de su dirección (aumento o disminución). Es preciso señalar que todos los ingresos son deflactados por el índice de precios al consumidor correspondiente a cada país de modo tal que en todo momento trabajamos con ingresos reales, es decir, ajustados por inflación.

Las encuestas de hogares de México y Brasil solo relevan ingresos laborales, de modo que en esos países solo es posible utilizar este tipo de ingresos en los cálculos. Para preservar la comparabilidad, los cálculos realizados en los demás países son reproducidos eliminando los ingresos no laborales del total, sin que esto arroje diferencias relevantes en los resultados.

\section{LA IMPORTANCIA DE LOS MOVIMIENTOS DEMOGRÁFICOS EN LA MOVILIDAD DE INGRESOS}

\section{III.1. Movimientos demográficos: quiénes entran y quiénes salen}

La tabla 1 presenta estadísticas sobre entradas y salidas de miembros en los hogares del panel analizado para cada país. En términos generales, la composición de los hogares parece ser estable en el tiempo, ya que la proporción de hogares que no experimentan movimientos demográficos oscila en torno al $75 \%$ del total. Sin embargo, la frecuencia e intensidad de este tipo de movimientos presenta diferencias relevantes entre los países analizados. Argentina y Brasil muestran la mayor estabilidad: la cantidad de miembros no varía en más del $80 \%$ de los casos, mientras que aumentos o reducciones considerables del tamaño del hogar (entrada o salida neta de 2 miembros o más) son muy poco frecuentes. En el extremo opuesto encontramos a Paraguay y a Perú, donde los movimientos demográficos resultan sensiblemente más frecuentes, afectando a más del $30 \%$ de los hogares y con una proporción mayor de movimientos de 2 o más miembros en términos netos. 
La proporción de entradas y salidas es similar en todos los países a excepción de Brasil y Paraguay, donde se registra una mayor proporción de salidas. Esto no responde a una diferencia estructural sino a decisiones metodológicas propias del relevamiento, ya que las encuestas de esos países no contienen información sobre niños menores de 10 años. Dado que, como veremos luego, las entradas de miembros en general incluyen una proporción importante de niños (lo cual es lógico debido a nacimientos), es natural que estas aparezcan subrrepresentadas en esos casos.

Los movimientos demográficos no pueden ser considerados intrínsecamente positivos o negativos en términos del bienestar de un hogar. Sin embargo, sí es posible plantear la hipótesis de que al menos algunos de ellos pueden correlacionarse con características negativas o eventos con consecuencias negativas sobre el hogar. Por ejemplo, los nacimientos pueden ser más frecuentes en hogares con nivel educativo más bajo debido al menor acceso a información sobre métodos de planificación familiar. De igual manera, los movimientos migratorios pueden estar correlacionados con el capital humano de los miembros de un hogar si este determina la probabilidad de inserción en determinadas ramas productivas. Por otra parte, como se muestra en Beccaria et al. (2015), la inestabilidad del ingreso es mayor en los hogares con menor nivel educativo y esto podría ser en sí mismo un factor determinante de movimientos demográficos, especialmente si los agentes toman ciertas decisiones sobre la composición de sus hogares como medio para influir sobre su flujo futuro de ingresos, como sugieren Duncan y Morgan. Por estos motivos, resulta de interés determinar si existe evidencia de correlación entre algunos movimientos demográficos y las características del hogar.

La tabla 2 presenta el tamaño medio, ingreso per cápita y nivel educativo del jefe de hogar para 3 grupos de hogares: aquellos que no registran entradas ni salidas, aquellos que registran entradas y aquellos que registran salidas (si bien estas últimas no son mutuamente excluyentes, los datos de la tabla 1 indican que la intersección entre ellas es pequeña). En primer lugar, se observa que los hogares donde entran miembros no tienen ex ante un tamaño diferente del de los hogares que no registran movimientos; en cambio, las salidas de miembros sí ocurren típicamente en hogares más grandes, lo cual es razonable. Las salidas de miembros en hogares grandes podrían responder a una demanda por mayor espacio habitacional o bien a la formación de nuevos núcleos familiares, posiblemente a partir del matrimonio de individuos jóvenes. También es posible que a partir de cierto tamaño las economías de escala al interior del hogar se agoten o incluso se reviertan, lo cual proveería incentivos a que los individuos abandonen el hogar para conformar uno nuevo. 
Tabla 1. Proporción de entradas y salidas y cambio en cantidad de miembros

\begin{tabular}{|c|c|c|c|c|c|c|c|}
\hline & Argentina & Brasil & Costa Rica & Ecuador & México & Paraguay & Perú \\
\hline \multicolumn{8}{|l|}{$\begin{array}{l}\text { Proporción de } \\
\text { entradas y salidas }\end{array}$} \\
\hline $\begin{array}{r}\text { Hogares que } \\
\text { registran entradas }\end{array}$ & $10.4 \%$ & $6.2 \%$ & $11.4 \%$ & $10.3 \%$ & $15.5 \%$ & $16.8 \%$ & $18.6 \%$ \\
\hline $\begin{array}{r}\text { Hogares que } \\
\text { registran salidas }\end{array}$ & $10.5 \%$ & $9.3 \%$ & $12.0 \%$ & $8.7 \%$ & $14.5 \%$ & $25.4 \%$ & $20.4 \%$ \\
\hline $\begin{array}{r}\text { Hogares que } \\
\text { registran ambas }\end{array}$ & $2.0 \%$ & $0.5 \%$ & $0.1 \%$ & $0.0 \%$ & $4.3 \%$ & $8.0 \%$ & $7.4 \%$ \\
\hline \multicolumn{8}{|l|}{$\begin{array}{r}\text { Cambio en la } \\
\text { cantidad de } \\
\text { miembros }\end{array}$} \\
\hline $\begin{array}{r}\text { Aumenta } \\
\text { en } 2 \text { o más }\end{array}$ & $2.1 \%$ & $1.4 \%$ & $2.7 \%$ & $2.7 \%$ & $4.8 \%$ & $2.2 \%$ & $3.9 \%$ \\
\hline Aumenta en 1 & $6.9 \%$ & $4.3 \%$ & $8.6 \%$ & $7.6 \%$ & $8.5 \%$ & $7.4 \%$ & $9.8 \%$ \\
\hline $\begin{array}{r}\text { Se mantiene } \\
\text { constante }\end{array}$ & $82.0 \%$ & $85.4 \%$ & $76.7 \%$ & $81.0 \%$ & $75.4 \%$ & $74.2 \%$ & $70.8 \%$ \\
\hline Baja en 1 & $6.6 \%$ & $6.8 \%$ & $8.0 \%$ & $5.7 \%$ & $6.8 \%$ & $12.1 \%$ & $9.7 \%$ \\
\hline Baja en 2 o más & $2.5 \%$ & $2.0 \%$ & $4.0 \%$ & $3.1 \%$ & $4.5 \%$ & $4.2 \%$ & $5.8 \%$ \\
\hline
\end{tabular}

Fuente: elaboración propia sobre la base de encuestas de hogares (ver sección 3)

Por otra parte, no encontramos evidencia concluyente de que los hogares que registran entradas de miembros disfruten de niveles de bienestar menores que aquellos que no sufren movimientos. El ingreso per cápita de los primeros es menor en algunos casos y mayor en otros, sin un patrón claro, aunque en todos los casos excepto en Perú se observa en estos un menor nivel educativo, lo que indicaría menor acumulación de capital humano, habitualmente correlacionado con la riqueza o el ingreso intertemporal del hogar. Un nivel de ingreso intertemporal bajo puede funcionar como incentivo a incorporar miembros al hogar con el fin de aprovechar economías de escala no explotadas al interior del mismo, lo que explicaría la correlación observada. En cambio, cuando se analizan los hogares que experimentan salidas de miembros, sí se obtiene un panorama más claro. En todos los casos, estos hogares tienen un ingreso per cápita medio menor y un perfil educativo peor que los hogares que no sufren movimientos.En otras palabras, existe cierto grado de correlación entre la salida de miembros de un hogar y características que afectan negativamente su bienestar. 


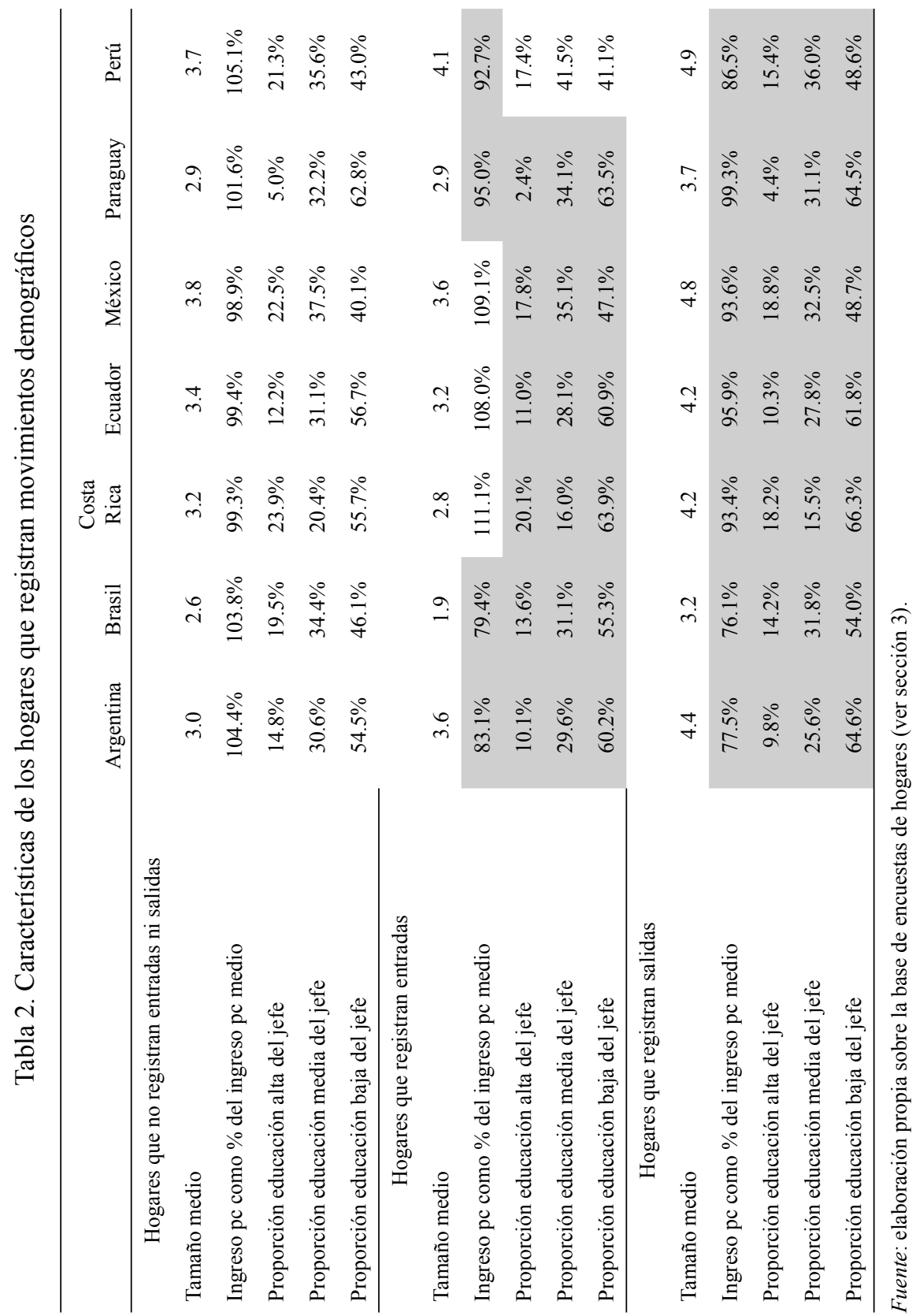




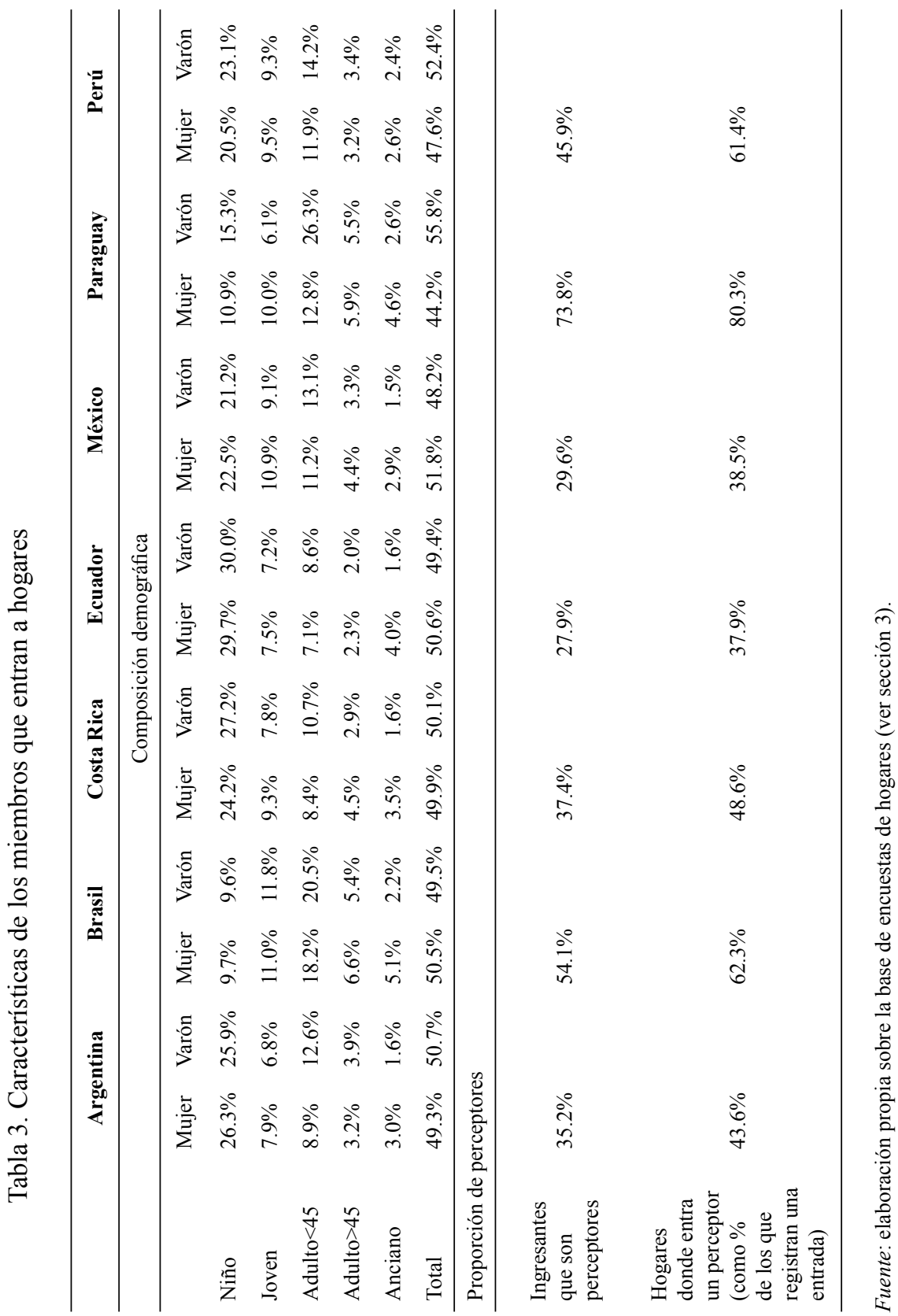


Esto podría ser consecuencia de movimientos migratorios originados en la necesidad de aumentar el ingreso total del hogar (especialmente en países donde el ingreso por remesas tiene un peso relevante). Alternativamente, la causalidad podría operar en sentido inverso: el bajo nivel de capital humano o de ingreso podrían ser consecuencia de un determinado nivel de inestabilidad en el núcleo del hogar.

El siguiente grupo etario en importancia es el de los adultos jóvenes, con un peso de entre $20 \%$ y $25 \%$ (lógicamente, mayor en Brasil y Paraguay). Al interior de ese grupo sí se observa mayor frecuencia de varones por sobre mujeres. Recordando que la tasa de actividad es mayor en hombres que en mujeres, esto sugiere cierta preferencia por el ingreso de individuos ocupados que perciben ingresos. De hecho, la proporción de ingresantes perceptores correlaciona positivamente con la diferencia a favor de varones en la proporción de ingresantes de esta franja etaria, lo cual respalda la hipótesis. También es importante notar que, en el subconjunto de hogares que registra ingresos de miembros, la proporción que recibe al menos un perceptor nuevo es en general muy alta (entre $38 \%$ y $80 \%$ ). Por su parte, los jóvenes de entre 18 y 25 años tienen cierto peso en la distribución de miembros que ingresan aunque marcadamente menor al de las categorías anteriores. Finalmente, el ingreso de adultos mayores o ancianos es muy poco frecuente.

Para complementar el análisis anterior, la tabla 4 presenta información análoga para aquellos miembros que salen de hogares en la ventana de observación. Resulta más difícil caracterizar de manera general estos movimientos dado que no emergen patrones claros. Sí podemos afirmar que la composición por género es nuevamente equilibrada, a excepción de la Argentina y Paraguay donde la proporción de varones es mayor entre los miembros salientes. Sin embargo, en lo que concierne a la composición por grupo etario, es difícil detectar generalidades. Niños, jóvenes y jóvenes adultos son las categorías más relevantes aunque el orden y la relación de proporciones entre ellas varían considerablemente de un país a otro. Los jóvenes adultos son la categoría más frecuente entre miembros salientes en todos los casos a excepción de Costa Rica y Ecuador; la proporción de estos casos ronda el $31 \%$, aunque alcanza el $41 \%$ en Brasil. Sin embargo, un hallazgo inesperado es la elevada frecuencia de salida de niños en todos los países analizados, aun en Brasil y Paraguay. La proporción de niños entre los miembros salientes oscila entre $16 \%$ y $49 \%$, siendo la categoría de mayor peso en Costa Rica y Ecuador, es decir, precisamente allí donde se dan menos salidas de adultos jóvenes. La salida de jóvenes tampoco es despreciable: comprende a una proporción de entre $17 \%$ y $28 \%$ de los casos.

La salida de jóvenes o adultos jóvenes puede ser producto de movimientos migratorios orientados a acceder a oportunidades laborales particulares. Por otro lado, también puede responder a decisiones de los miembros de formar hogares nue- 
vos, factiblemente a través de matrimonios. Ninguna de las dos posibilidades anteriores es incompatible con la salida de niños, que a su vez puede ser consecuencia de la disolución de hogares debida a divorcios o separaciones. Sin embargo, la elevada frecuencia de la salida de niños definitivamente contradice la intuición y sugiere considerar la posibilidad de que existan problemas de captación en estas encuestas. Por último, las salidas de adultos mayores y ancianos tienen cierta frecuencia (tomadas conjuntamente, representan entre un $11 \%$ y un $27 \%$ de los casos), parcialmente atribuible a defunciones.

Dado que las salidas involucran miembros de mayor edad que las entradas, es de esperar que también involucren más frecuentemente a perceptores de ingresos. De hecho, la proporción de salientes que percibe ingresos es siempre superior a la proporción de entrantes que percibe ingresos. En consecuencia, entre un $52 \%$ y un $82 \%$ de los hogares que registraron salidas de miembros sufrieron la pérdida de al menos un perceptor de ingresos. Estos datos deben ser puestos en el contexto que sugiere el análisis de la tabla 2, donde observamos que las salidas son más frecuentes en hogares de ingreso y nivel educativo más bajos. La correlación entre salida de miembros y peores condiciones socioeconómicas, por un lado, y la elevada proporción de perceptores de ingresos entre los miembros salientes, por el otro, indican que este subconjunto de hogares probablemente sufre de una inestabilidad de ingresos elevada, con consecuencias negativas para su bienestar. Analizamos con mayor profundidad este punto en la subsección siguiente.

III.2. Contribución de los movimientos demográficos a la movilidad de ingresos

En Beccaria et al. (2015) se cuantifica el tamaño de la movilidad de ingresos para estos países y se utiliza la metodología de microsimulación para separar la contribución de sus diversos componentes. En este trabajo continuamos esa línea de análisis aplicando la misma metodología para cuantificar la contribución de los movimientos demográficos a la movilidad total observada. Para eso, imputamos ingresos a aquellos individuos que entran o salen en el segundo período de observación, de acuerdo con las siguientes reglas:

$$
\begin{gathered}
y_{i 2}=y_{i 1} \cdot \frac{\bar{y}_{2}}{\bar{y}_{1}} \text { si } y_{i 1} \text { es observado pero } y_{i 2} \text { no (salida) } \\
y_{i 1}=y_{i 2} \cdot \frac{\bar{y}_{1}}{\bar{y}_{2}} \text { si } y_{i 2} \text { es observado } \\
\text { pero } y_{i 1} \text { no (entrada) }
\end{gathered}
$$




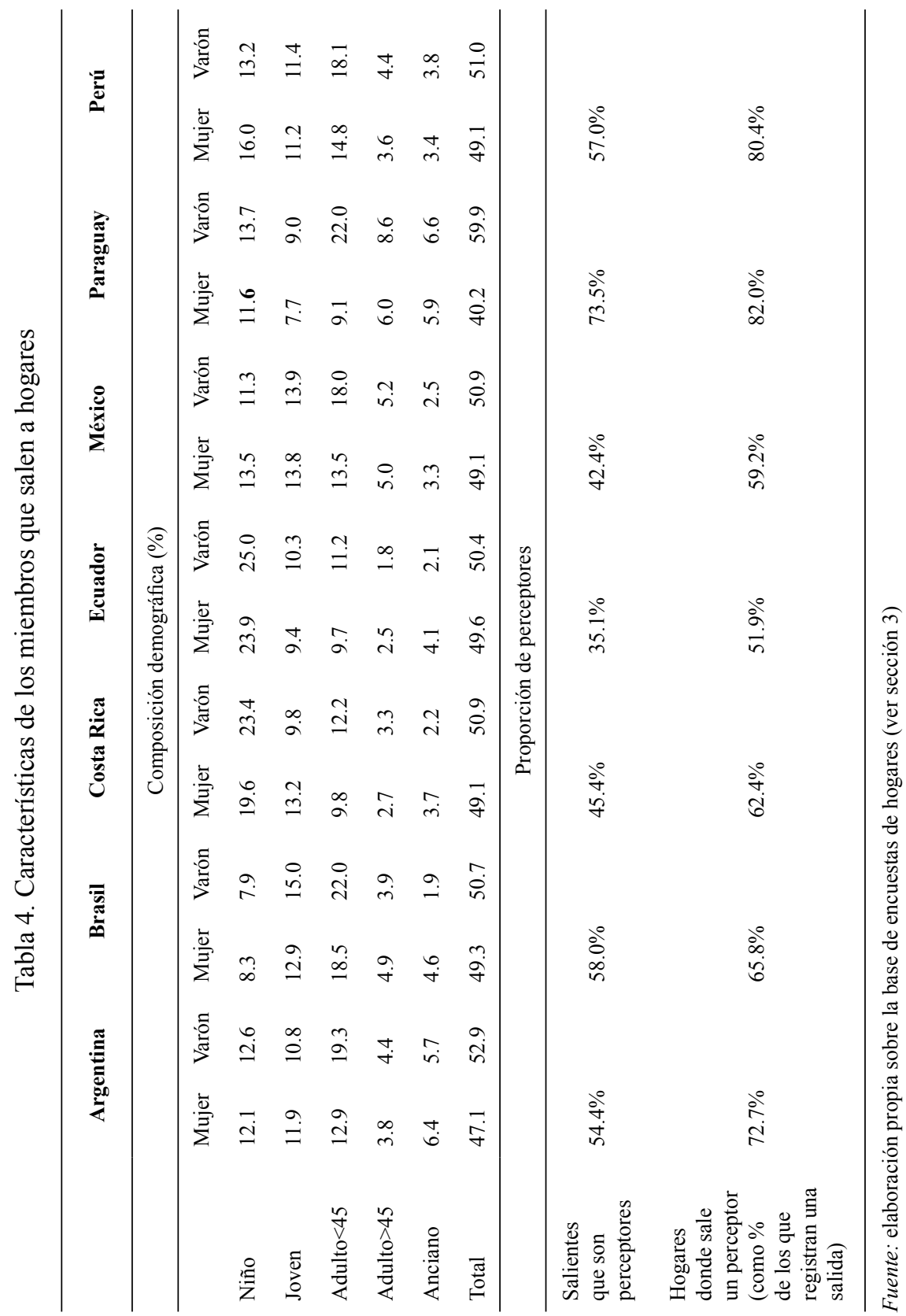


El objetivo de este ejercicio consiste en computar el valor que adoptaría el indicador si los movimientos demográficos no existieran y cada individuo que entra o sale mantuviera el ingreso observado del período en que sí es relevado, corregido por el cambio medio en los ingresos entre períodos. Los resultados de este ejercicio se presentan en la tabla 5 junto con el cálculo anterior de movilidad para la muestra total y para algunas submuestras de interés. Debe tenerse en cuenta, como se detalló en la sección 3, que en Brasil y México solo el ingreso laboral es observado, aunque esta limitación no tiene gran incidencia en estos resultados.

Tabla 5. Movilidad de ingresos observada y microsimulación sin movimientos demográficos

Argentina Brasil* Costa Rica México* Ecuador Paraguay Perú

\begin{tabular}{|c|c|c|c|c|c|c|c|}
\hline \multicolumn{8}{|c|}{ Movilidad observada } \\
\hline Total & 0.40 & 0.34 & 0.45 & 0.56 & 0.52 & 0.50 & 0.51 \\
\hline $\begin{array}{l}\text { Hogares que } \\
\text { registran } \\
\text { entradas }\end{array}$ & 0.54 & 0.58 & 0.61 & 0.65 & 0.59 & 0.65 & 0.62 \\
\hline $\begin{array}{l}\text { Hogares que } \\
\text { registran salidas }\end{array}$ & 0.58 & 0.56 & 0.68 & 0.71 & 0.61 & 0.60 & 0.65 \\
\hline $\begin{array}{l}\text { Hogares que } \\
\text { registran ambas }\end{array}$ & 0.55 & 0.57 & 0.60 & 0.66 & 0.59 & 0.62 & 0.60 \\
\hline \multicolumn{8}{|c|}{ Simulación } \\
\hline $\begin{array}{l}\text { Movilidad no } \\
\text { demográfica }\end{array}$ & 0.36 & 0.30 & 0.40 & 0.50 & 0.45 & 0.35 & 0.43 \\
\hline $\begin{array}{l}\text { Proporción } \\
\text { de movilidad } \\
\text { demográfica }\end{array}$ & $9.2 \%$ & $11.1 \%$ & $12.4 \%$ & $10.7 \%$ & $13.2 \%$ & $28.6 \%$ & $16.3 \%$ \\
\hline
\end{tabular}

*Ingreso laboral

Fuente: elaboración propia sobre la base de encuestas de hogares (ver sección 3).

En el primer panel de la tabla 5 podemos observar el grado de inestabilidad de ingresos en tres submuestras de interés: hogares que registran entradas de miembros, hogares que registran salidas de miembros y hogares que registran ambas. Los hogares que registran salidas presentan la mayor inestabilidad de ingresos en todos los casos a excepción de Brasil y Paraguay, donde de todos modos esta es marcadamente mayor que en la muestra general. Se confirma de este modo la idea de que los hogares que sufren la pérdida de alguno de sus miembros están caracterizados por una volatilidad especialmente elevada en su flujo de ingresos, diferencia que 
es particularmente grande en la Argentina, Brasil y Costa Rica, donde promedia el $52 \%$. Los hogares que experimentan una incorporación de nuevos miembros también presentan valores más altos que la muestra general, aunque en la mayoría de los casos la diferencia relativa es de menor magnitud. Queda claro entonces que los movimientos demográficos constituyen una fuente relevante de variabilidad de los ingresos para al menos un subconjunto de los hogares de los países analizados.

El segundo panel muestra los resultados del ejercicio de microsimulación. Naturalmente, la inestabilidad de ingresos resulta menor si se anulan los movimientos demográficos, pero la magnitud de esta reducción no es evidente $a$ priori. De acuerdo con el procedimiento realizado, podemos observar que esta resulta ser pequeña pero no despreciable. La Argentina es el país donde menor es el peso de estos movimientos, ya que la reducción obtenida es de solo $9.2 \%$, mientras que el extremo opuesto es Paraguay con un $28.6 \%$. En los demás países, el cambio se ubica en la franja de entre $10 \%$ y $20 \%$.

Resulta claro que los movimientos demográficos no tienen la misma relevancia que las entradas y salidas de miembros del hogar al mercado de trabajo, sin embargo, sí resulta similar en varios casos al peso de las fluctuaciones en los ingresos no laborales de los hogares (ver Beccaria et al., 2015). Este hallazgo puede ser interpretado en diversos sentidos. Por un lado, puede resultar desalentador que movimientos de tipo demográfico, muchos de los cuales factiblemente escapan al ámbito de influencia de las políticas públicas, introduzcan tal nivel de volatilidad en el flujo de ingresos de los hogares. Alternativamente, se puede resaltar como un resultado positivo que las transferencias de origen público en su magnitud actual (en la medida en que representan la mayor parte de los ingresos no laborales de los hogares) sean potencialmente capaces de compensar esta inestabilidad de origen demográfico. La factibilidad de ese objetivo y sus consecuencias sociales deberían ser materia de investigación futura.

Una transformación adecuada de la variable de interés podría ser útil para enriquecer nuestra comprensión del problema: podemos emplear el ingreso per cápita del hogar en lugar del total para incorporar el hecho de que hogares con distintos tamaños tienen distintas necesidades. La tabla 6 presenta los resultados de repetir el ejercicio anterior calculando a partir del ingreso per cápita (ya sea observado o simulado).

Diversos aspectos de la tabla 6 son relevantes. En primer lugar, cabe señalar que la movilidad observada computada sobre el ingreso per cápita difiere en general muy poco de la computada a partir del ingreso total. La diferencia hallada es menor 
al $5 \%$ en todos los casos, a excepción de México, donde sí presenta un tamaño relevante. Esto podría atribuirse a características particulares de la distribución del ingreso en ese país o bien a las propiedades técnicas de la medida utilizada. En cualquier caso, encontramos que en la mayoría de los países la elección entre una u otra definición del ingreso de interés es poco relevante para nuestros fines.

Tabla 6. Movilidad de ingresos observada y microsimulación sin movimientos demográficos (ingreso per cápita)

\begin{tabular}{|c|c|c|c|c|c|c|c|}
\hline & Argentina & Brasil* & Costa Rica & México* & Ecuador & Paraguay & Perú \\
\hline \multicolumn{8}{|c|}{ Movilidad observada } \\
\hline Total & 0.40 & 0.34 & 0.44 & 0.57 & 0.51 & 0.47 & 0.50 \\
\hline $\begin{array}{l}\text { Hogares que } \\
\text { registran entradas }\end{array}$ & 0.52 & 0.54 & 0.57 & 0.68 & 0.62 & 0.59 & 0.59 \\
\hline $\begin{array}{l}\text { Hogares que } \\
\text { registran salidas }\end{array}$ & 0.54 & 0.53 & 0.61 & 0.70 & 0.65 & 0.53 & 0.60 \\
\hline $\begin{array}{l}\text { Hogares que } \\
\text { registran ambas }\end{array}$ & 0.52 & 0.53 & 0.57 & 0.68 & 0.61 & 0.56 & 0.57 \\
\hline \multicolumn{8}{|c|}{ Simulación } \\
\hline $\begin{array}{l}\text { Movilidad no } \\
\text { demográfica }\end{array}$ & 0.40 & 0.36 & 0.46 & 0.58 & 0.53 & 0.42 & 0.50 \\
\hline $\begin{array}{l}\text { Relación no } \\
\text { demográfica/ } \\
\text { observada }\end{array}$ & $102.1 \%$ & $107.6 \%$ & $102.9 \%$ & $101.3 \%$ & $103.5 \%$ & $89.7 \%$ & $99.6 \%$ \\
\hline *Ingreso laboral & & & & & & & \\
\hline
\end{tabular}

Fuente: elaboración propia sobre la base de encuestas de hogares (ver sección 3).

En segundo lugar, la mayor inestabilidad del ingreso en hogares que experimentan cambios demográficos se mantiene, aunque la magnitud de las diferencias se reduce ligeramente, lo cual es razonable en la medida en que una parte del impacto sobre el ingreso es amortiguado por el cambio en la cantidad de miembros del hogar. También encontramos, en general, los valores de volatilidad más altos en los hogares que sufren salidas de miembros, con las mismas excepciones que antes (Brasil y Paraguay). En cambio, los resultados del ejercicio de microsimulación sí presentan cambios sustanciales. El ingreso per cápita es naturalmente mucho menos sensible a los cambios demográficos que el ingreso total, por lo tanto, el peso de estos movimientos necesariamente debe reducirse al utilizar esta nueva medida. 
Que la movilidad calculada a partir del ingreso total y la calculada a partir del ingreso per cápita sean muy similares, incluso en hogares que registran salidas y entradas de miembros, es un resultado central que amerita un análisis más profundo. De acuerdo con lo explicado en la primera parte de la sección anterior, sabemos que un porcentaje relevante de miembros entrantes y salientes son perceptores de ingresos, por lo tanto no es extraño que el ingreso total y el ingreso per cápita presenten movimientos aproximadamente similares. A su vez, esto es consistente con la hipótesis de que los hogares buscan suavizar el flujo de ingreso per cápita en el tiempo, ya que los movimientos demográficos podrían estar condicionados por este objetivo. Sin embargo, también es frecuente el ingreso o salida de miembros que no perciben ingresos, lo cual naturalmente tiene efectos sobre el ingreso del hogar. Por ejemplo, el ingreso de un nuevo miembro que no percibe ingresos al hogar equivale, ceteris paribus, a una reducción en el ingreso per cápita del mismo. Sin embargo, si esto incentivara a otros miembros a aumentar sus ingresos por alguna vía, el ingreso per cápita podría reducirse en menor medida, mantenerse constante o incluso aumentar. En otras palabras, es posible que existan estrategias al interior del hogar que apunten a compensar el efecto de los movimientos demográficos sobre el ingreso total.

Dado que típicamente los individuos no tienen mucho control sobre el flujo de ingresos no laborales que reciben, es natural pensar que las estrategias a las que nos referimos consisten en entrar o salir del mercado de trabajo, cambiar de un empleo a otro o modificar la cantidad de horas trabajadas. En otras palabras, conjeturamos que el ingreso laboral del hogar puede ajustarse ante cambios en su composición. En la siguiente sección, exploramos a fondo esta hipótesis y proponemos diversos ejercicios de estimación que proveen evidencia que la respalda en uno de los países de la muestra.

\section{ESTRATEGIAS DEL HOGAR}

En esta sección, proveemos evidencia de la existencia de estrategias como las descriptas anteriormente para el caso de la Argentina. Por lo discutido anteriormente, resulta claro que una parte relevante de los movimientos demográficos son de naturaleza endógena, en el sentido de que persiguen un objetivo vinculado a la satisfacción de necesidades materiales de miembros del hogar. Esto impide el análisis de estrategias ya que dificulta la determinación de qué conducta puede ser interpretada como una reacción. Sin embargo, es posible conjeturar que algunos movimientos demográficos son exógenos en el sentido de que no responden a necesidades materiales sino a necesidades de otro tipo (emocionales, psicológicas, etcétera) o bien son accidentales. 
La llegada de un niño al hogar ${ }^{2}$ es un candidato natural a integrar este último grupo por varias razones. En primer lugar, porque es posible que no responda a intenciones de ninguno de los miembros del hogar. En segundo lugar, porque es inverosímil que responda a un objetivo vinculado a los ingresos. En consecuencia, asumiremos en lo sucesivo que se trata de un evento exógeno y analizaremos su influencia sobre la conducta de los demás miembros del hogar, que interpretaremos como una reacción (si efectivamente la hay) a la llegada de niños al hogar.

La hipótesis de la que partimos es que la llegada de un niño al hogar provee incentivos a aumentar la oferta de trabajo en los miembros del hogar que están en edad activa. Este ajuste puede darse en el margen extensivo, en el margen intensivo o en ambos, aunque centraremos la discusión sobre el margen extensivo por tratarse de aquel que ofrece resultados más interesantes. Para testear esta hipótesis, realizamos diversos ejercicios de estimación de un modelo donde la variable independiente es una dummy que indica la llegada de un niño al hogar en un período y la variable independiente es una dummy que indica el ingreso al empleo de algún miembro del hogar previamente no ocupado. Resulta de gran importancia distinguir el género del ingresante ya que, como veremos, los comportamientos difieren notablemente entre varones y mujeres.

Un panel de dos observaciones plantea ciertas limitaciones para un análisis de este tipo, en particular porque impide aplicar transformaciones que permitan aislar la incidencia de heterogeneidades inobservables entre hogares. Por ese motivo, explotamos más intensamente el esquema rotativo particularmente de la encuesta argentina y construimos paneles de cuatro observaciones para todo el período 2003-2015 en ese país. En esa base, construimos las variables de interés y también variables de control de uso habitual en la literatura: dummies que indican el nivel educativo del jefe del hogar, edad del jefe del hogar, dummies temporales (por año y por trimestre), dummies regionales y una dummy que indica si el hogar ya contaba con niños antes de la llegada del nuevo integrante.

A continuación, procedemos a estimar la regresión de interés mediante tres estimadores de uso habitual: Mínimos Cuadrados Ordinarios (MCO), Efectos Aleatorios (EA) y Efectos Fijos (EF). Recordemos que la diferencia fundamental es que el tercero controla por heterogeneidades inobservables mientras que los primeros dos no. Los resultados, para distintas variables dependientes, aparecen resumidos en la tabla 7 .

2 Las encuestas de hogares utilizadas no permiten distinguir si estas llegadas son fruto de nacimientos o de otros movimientos. Si bien consideramos la primera posibilidad como mucho más factible, no se trata de un punto relevante para los fines del análisis que aquí emprendemos. 
Tabla 7. Estrategias del hogar frente a la llegada de un niño: entradas

\begin{tabular}{lccc}
\hline \multicolumn{1}{c}{ Variable dependiente } & Media muestral & Estimador & Resultado \\
\hline Dummy al menos una entrada al empleo & & MCO & $0.039^{* * *}$ \\
Dummy al menos una entrada al empleo & 0.0860 & EA & $0.037^{* * *}$ \\
Dummy al menos una entrada al empleo & & EF & $0.010^{*}$ \\
\hline Dummy al menos un hombre entra al empleo & \multirow{2}{*}{0.0403} & ECO & $0.028^{* * *}$ \\
Dummy al menos un hombre entra al empleo & & EF & $0.027^{* * *}$ \\
Dummy al menos un hombre entra al empleo & & MCO & $0.013^{* * *}$ \\
\hline Dummy al menos una mujer entra al empleo & \multirow{2}{*}{0.0505} & EA & $0.017^{* * * *}$ \\
Dummy al menos una mujer entra al empleo & & EF & -0.002 \\
\hline Dummy al menos una mujer entra al empleo & & &
\end{tabular}

Fuente: elaboración propia.

El primer panel presenta los resultados de la regresión inicialmente propuesta. La estimación por MCO o por EA arroja un resultado estadísticamente significativo y de tamaño relevante (casi la mitad de la media muestral observada), lo que indica que efectivamente la llegada de un niño al hogar produce un aumento importante en la probabilidad de que un miembro no ocupado del mismo entre al mercado de trabajo. Sin embargo, cuando se aplica el estimador de EF para eliminar el posible efecto de heterogeneidades inobservables, este efecto desaparece por completo. En otras palabras, si bien es cierto que en muchos hogares la llegada de niños aparece asociada al ingreso de miembros al empleo, no se trata de un efecto causal sino de correlación. Son otras características del hogar las que motivan el movimiento observado. Es importante señalar que el hecho de que el efecto no sea causal no implica que de hecho no exista la compensación explicada entre el movimiento demográfico y el del ingreso total del hogar; sí indica que no es posible atribuir a esta una interpretación de tipo causal.

Es un hallazgo habitual en la literatura que las decisiones de oferta laboral varían considerablemente entre hombres y mujeres, en particular en lo que respecta a la influencia de la cantidad de hijos. Para analizar esta posibilidad en nuestro modelo, los dos paneles inferiores de la tabla 7 permiten descomponer el efecto anterior en lo que ocurre con varones y mujeres por separado. Los resultados son marcadamente diferentes. Para el caso de miembros de sexo masculino, existe un efecto causal de 1,3 puntos porcentuales (alrededor de un tercio de la media observada) sobre la probabilidad de entrar al mercado de trabajo como consecuencia de 
la llegada de un niño al hogar. Por el contrario, en los miembros de sexo femenino no se observa un efecto causal, mientras que el efecto no causal obtenido por MCO y EA es mucho más pequeño en tamaño.

Estos resultados indican que la llegada de niños al hogar motiva el ingreso de miembros varones al mercado de trabajo pero no así de miembros mujeres. Este hallazgo es consistente con la abundante literatura que documenta el mayor peso del trabajo de cuidado (o del trabajo no remunerado en el hogar en general) en las mujeres con respecto a los varones. Asimismo, si tenemos en cuenta que existe una brecha de ingresos relevante entre los salarios de hombres y de mujeres, la entrada de varones al empleo es una estrategia racional en relación con el objetivo de obtener ingresos que compensen la presencia de un nuevo miembro. De hecho, precisamente por estos motivos, es posible que las mujeres tengan incentivos a retirarse del mercado de trabajo ante la llegada de niños al hogar, de modo que el mecanismo operaría en sentido inverso. Para investigar esta posibilidad con mayor profundidad, reproducimos el ejercicio anterior pero invirtiendo el sentido de la variable dependiente: ahora utilizamos una dummy que indica la salida del empleo por parte de un miembro del hogar.

La tabla 8 presenta los resultados de estas nuevas estimaciones. A diferencia del ejercicio anterior, aquí sí encontramos evidencia de mayor probabilidad de salida del mercado de trabajo por parte de algún miembro del hogar causada por la llegada de un niño al hogar, luego de controlar por heterogeneidades inobservables. El tamaño de este efecto es de 1,9 puntos porcentuales, casi la cuarta parte de la media observada, de modo que se trata de un aumento considerable. Como se ve, las decisiones de oferta de trabajo al interior del hogar son sensibles a movimientos demográficos en más de un sentido.

Nuevamente el análisis se enriquece cuando se lo divide por género. En varones, el efecto de la llegada de niños sobre la salida de miembros existe y tiene un tamaño relevante cuando se lo estima por $\mathrm{MCO}$ o EA, pero desaparece cuando se lo estima por EF. En otras palabras, se trata de otro caso de un efecto no causal. No podemos afirmar que los varones tengan ceteris paribus mayor probabilidad de abandonar sus puestos de trabajo debido a la llegada de niños al hogar, aunque ese comportamiento se observa en muchos casos luego de controlar por otros factores observables. En cambio, para las mujeres, sí se obtiene un efecto causal por EF. De hecho, el tamaño de este efecto es casi idéntico al obtenido en la regresión general, pero dado que la media observada es menor para mujeres, su peso relativo se vuelve mayor (ligeramente por encima de un tercio). 
Tabla 8. Estrategias del hogar frente a la llegada de un niño: salidas

\begin{tabular}{lccc}
\hline \multicolumn{1}{c}{ Variable dependiente } & Media muestral & Estimador & Resultado \\
\hline Dummy al menos una salida del empleo & & MCO & $0.059 * * *$ \\
Dummy al menos una salida del empleo & 0.0837 & EA & $0.057^{* * *}$ \\
Dummy al menos una salida del empleo & & EF & $0.020^{* * *}$ \\
\hline Dummy al menos un hombre sale del empleo & & MCO & $0.015^{* * *}$ \\
Dummy al menos un hombre sale del empleo & 0.0370 & EA & $0.014^{* * *}$ \\
Dummy al menos un hombre sale del empleo & & EF & -0.001 \\
\hline Dummy al menos una mujer sale del empleo & & MCO & $0.046^{* * *}$ \\
Dummy al menos una mujer sale del empleo & 0.0509 & EA & $0.046^{* * *}$ \\
Dummy al menos una mujer sale del empleo & & EF & $0.019^{* * *}$ \\
\hline
\end{tabular}

Fuente: elaboración propia.

Tomados de conjunto, los ejercicios de estimación realizados indican que la llegada de niños al hogar causa el ingreso de varones y la salida de mujeres al mercado de trabajo. Las magnitudes de estos efectos no son muy distintas en términos absolutos ni en términos relativos, de modo que es posible que la cantidad de miembros ocupados a nivel general no se altere sensiblemente. Sin embargo, al nivel del hogar, sí es de esperar que el efecto sobre el ingreso laboral sea considerable, habida cuenta del diferencial existente entre los salarios de varones y de mujeres. De este modo, la reducción del ingreso per cápita experimentada por el ingreso de un niño se vería compensada (al menos parcialmente) por un aumento en el ingreso laboral del hogar, reduciendo así la inestabilidad de ingresos a la que el hogar se encuentra expuesto.

\section{CONCLUSIONES}

Este trabajo analiza el tamaño y las características de los movimientos demográficos en los hogares de siete países latinoamericanos para el período 20032015. Como explica el grueso de la literatura especializada, estos movimientos pueden ser de gran importancia en la determinación del sendero temporal de ingresos de los hogares. De acuerdo con la evidencia presentada aquí, es posible afirmar que los cambios de tamaño y composición de los hogares latinoamericanos son de magnitud y frecuencia pequeña pero relevante. Ejercicios de microsimulación indican que su contribución a la inestabilidad observada en los ingresos de los hogares no es despreciable. 
La llegada de niños al hogar es uno de los movimientos de mayor frecuencia y sus efectos sobre el ingreso per cápita del hogar son claramente negativos. En este trabajo proveemos evidencia de la existencia de estrategias al interior del hogar para compensar este efecto, de modo tal de reducir la volatilidad en el flujo de ingresos. En particular, nuestras estimaciones indican que la llegada de niños tiene un efecto positivo tanto sobre la entrada de varones al mercado de trabajo como sobre la salida de mujeres del mismo. Estos efectos pueden interpretarse tanto en términos de oferta laboral como de distribución del trabajo de cuidado al interior del hogar. Pero, fundamentalmente, pueden tener un efecto de reducción de la volatilidad del ingreso del hogar.

\section{BIBLIOGRAFIA}

Alves, N., \& Martins, C. (2014). Household income mobility in the European Union and in Portugal: an analysis of labor market and demographic events. Economic Bulletin and Financial Stability Report Articles, 53-63.

Bartels, C., \& Bonke, T. (2013). Can households and welfare states mitigate rising earnings instability? The review of income and wealth, 59(2), 250-282.

Beccaria, L., Maurizio, R., Trombetta, M., \& Vazquez, G. (2015). Income instability during a period of economic growth and improving social and labor conditions: Latin America in the 2000s. Trabajo presentado en la LI Reunión Annual de la Asociación Argentina de Economía Política. Recuperado de http://www.aaep.org.ar/anales/works/works2016/beccaria.pdf

Chiappori, P. (1992). Collective labor supply and welfare. Journal of Political Economy 100 (3), 437-467.

Duncan, G., \& Morgan, J. (1981). Persistence and change in economic status and the role of changing family composition. En M. Hill, D. Hill, \& J. Morgan, Five thousand American families - Patterns of economic progress (pp. 1-45). Ann Arbor: Institute for Social Research.

Fields, G., \& Ok, E. (1999). Measuring Movement of Incomes. Economica 66(264), 455-471

Fritzell, J. (1990). The dynamics of income distribution: Economic mobility in Sweden in comparison with the United States. Social Science Research 19(1), 17-46.

Gittleman, M., \& Joyce, M. (1999). Have familiy income mobility patterns changed? Demography 36(3), 299-314.

Jarvis, S., \& Jenkins, S. (1997). Marital splits and income changes: evidences for Britain. Innocenti Economic and Social Policy Series 60, UNICEF. 
(C) 2017 por los autores; licencia otorgada a la revista Estudios económicos. Este artículo es de acceso abierto y distribuido bajo los términos y condiciones de una licencia Atribución-No Comercial 3.0 Unported (CC BY-NC 3.0) de Creative Commons. Para ver una copia de esta licencia, visite http://creativecommons.org/ licenses/by-nc/3.0/ 